\title{
Stable aqueous aluminum-manganese photoelectrochemical cells with high-rate and high- efficiency abilities
}

Shuqiang Jiao ( $\square$ sjiao@ustb.edu.cn )

University of Science and Technology Beijing

\section{Xuefeng Zhang}

University of Science and Technology Beijing

\section{Wei-li Song}

Beijing Institute of Technology

\section{Mingyong Wang}

University of Science and Technology Beijing https://orcid.org/0000-0002-5246-163X

Jiguo Tu

University of Science and Technology Beijing

Handong Jiao

University of Science and Technology Beijing

\section{Article}

Keywords: aqueous aluminum-ion batteries (AAIBs),photoelectrochemical cells, energy density, charge

Posted Date: July 22nd, 2021

DOI: https://doi.org/10.21203/rs.3.rs-714704/v1

License: (c) (1) This work is licensed under a Creative Commons Attribution 4.0 International License.

Read Full License 
Stable aqueous aluminum-manganese photoelectrochemical cells with high-rate and high-efficiency abilities

Xuefeng Zhang, ${ }^{1}$ Wei-Li Song, ${ }^{2}$ Mingyong Wang, ${ }^{1 *}$ Jiguo Tu, ${ }^{1}$ Handong Jiao, ${ }^{2}$ Shuqiang $\mathrm{Jiao}^{1 *}$

${ }^{1}$ State Key Laboratory of Advanced Metallurgy, University of Science and Technology Beijing, Beijing 100083, P. R. China. E-mail: sjiao@ustb.edu.cn (S. Jiao), mywang@ustb.edu.cn (M. Wang)

${ }^{2}$ Institute of Advanced Structure Technology, Beijing Institute of Technology, Beijing 100081, P R China. E-mail: weilis@ bit.edu.cn (W.-L. Song) 


\begin{abstract}
Aqueous aluminum-ion batteries (AAIBs) are potential candidates for large-scale energy storage devices for their advantages of high energy density, resource abundance, low cost, safety, and environmental friendliness. Due to various redox procedures, good reversibility, and high discharge potential, the aqueous aluminum-manganese oxide battery has drawn wide attention, while the critical issues induced from slow kinetics and undesired soluble $\mathrm{Mn}^{2+}$ lead to slow charging, poor rate capability, and low energy density. However, there is very limited progress for performance improvement via conventional chemical or physical modification approaches. To overcome these challenges, an efficient photo-regulation strategy has been proposed in terms of direct radiating visible light on the cell during the galvanostatic charging and discharging. The efficient separation and transmission of photoelectrons in the photo positive electrode dramatically improves the dynamics, and fast charging and enhanced rate performance could be achieved. Photo-oxidation behavior can effectively promote the conversion of soluble $\mathrm{Mn}^{2+}$, thus further enhancing the energy density of the as-assembled aluminummanganese battery. Furthermore, a photo-conversion efficiency of up to $1.2 \%$ has been acquired. Based on the photo-regulation strategy, the mechanism of the photoelectrochemical coupling system has been understood, which opens a promising route for achieving photoelectrochemical batteries with high energy density and fast charge.
\end{abstract}




\section{Introduction}

The increasing consumption of fossil fuels and critical environmental issues are required to develop eco-friendly and sustainable renewable energy resources and energy storage systems. Aluminum-ion batteries (AIBs) are potential candidates for large-scale energy storage due to the advantages of high energy density, resource abundance, low cost, and safety. However, the current non-aqueous halides ionic liquid electrolytes of high cost, severe corrosivity, air sensitivity, and environmental concerns have significantly restricted their practical applications ${ }^{1-3}$. In contrast, aqueous electrolytes with features of higher safety, lower cost, $\mathrm{H}_{2} \mathrm{O} / \mathrm{O}_{2}$ insensitivity, and easier fabrication has attracted remarkable attention ${ }^{4-6}$. Among such battery prototype, manganese oxides with layered structure and high energy density are widely employed as the positive electrode materials for assembling aqueous aluminum ion batteries (AAIBs). ${ }^{7-9}$ In particular, $\mathrm{Al}_{\mathrm{x}} \mathrm{MnO}_{2}$ phase is generated by the intercalation of $\mathrm{Al}^{3+}$ during the discharging process, and subsequently restored to the pristine $\alpha-\mathrm{MnO}_{2}$ phase upon a reversible electrochemical process (Supplementary, Fig. 1) ${ }^{7,10}$.

However, $\alpha-\mathrm{MnO}_{2}$ electrode still suffers from poor rate capability and slow charging because of its slow kinetics. Also, the generated soluble Mn-based compounds during the discharging process causes a low energy density and poor cycling stability ${ }^{11}$. Moreover, the presence of tight oxide film $\left(\mathrm{Al}_{2} \mathrm{O}_{3}\right)$ on the unmodified $\mathrm{Al}$ negative electrode surface would induce a large overpotential for plating/stripping of $\mathrm{Al}^{3+}$. Various improvement strategies based on chemical or physical approaches have been proposed to address the issues, e.g., the nano-scale design of active materials ${ }^{8}$, manganese ions $\left(\mathrm{Mn}^{2+}\right)$ electrolyte pre-addition strategy ${ }^{10}$, alloying of the negative 
electrode, and etc. ${ }^{7}$. Although a significant progress has been achieved, it is still a challenge to fulfill the a fast-charge battery system along with the ever-growing requirement of high energy density.

Noted that $\alpha-\mathrm{MnO}_{2}$ is an n-type semiconductor with a photoelectric response ability for its narrow bandgap $(\sim 1.3-2.5 \mathrm{eV})$, and thus visible light could be used to manipulate the electronic structure and valence stages ${ }^{12,13}$. Based on such characteristic, here we proposed a photo-regulation strategy by direct radiation of visible light during the galvanostatic charging and discharging, aiming to promoting the rate capability and energy density of the electrochemical battery. In the design of such photoelectrochemical cells, photo positive electrode is the essential component of photo-rechargeable batteries, which are required to possess unique features. (i) The designed photo positive electrodes should follow the principle of energy level matching, and whereby the photo-excited electrons can transport as expected. (ii) An optically transparent electrode coupled with current collector should be designed for receiving optical radiation. As a potential candidate, transparent substrates coated with indium tin oxide (ITO), fluorine-doped tin oxide (FTO) or porous electrode prototypes would be selected. (iii) Active materials of electrochemically stable microstructures along with both high electrochemical activity and light absorption capacity are also required. To implement such concept, in this contribution, the photo positive electrode was fabricated based on $\alpha-\mathrm{MnO}_{2}$ nanorod (as both the active material and photosensitizer), reduced graphene oxide (as the electron transport material), and porous carbon fiber (CF) current collector. In principle, the electron-hole pairs are generated when light 
radiates to the surface of the photosensitizers. Subsequently, the generated photoelectron would flow to the external circuit owing to the appropriate matched energy level. On the other hand, the photoexcited electrons can transport along the nanorods, which is beneficial to reduce the recombination rate of separated electronhole pairs. The exposure of $\alpha-\mathrm{MnO}_{2}$ to visible light could effectively lower the charge transport resistance, which is favorable to generate photooxidation behaviors. In the photoelectrochemical battery, consequently, the overall performance including fast recharging, enhanced rate performance, and suppressed soluble $\mathrm{Mn}^{2+}$ has been achieved.

\section{Results}

The cell configurations and operation principles. In this study, $\alpha-\mathrm{MnO}_{2}$ nanorod was synthesized using a simple solvothermal method, which was simultaneously employed as electrochemically active materials and photosensitizer. The chemical composition and elemental valence of the as-prepared $\alpha-\mathrm{MnO}_{2}$ were firstly verified by X-ray diffraction (XRD) and X-ray photoelectron spectroscopy (XPS) (Supplementary, Fig. 2A,B). Moreover, the scanning electron microscopy (SEM) and transmission electron microscopy (TEM) images revealed that the as-synthesized $\alpha-\mathrm{MnO}_{2}$ presented a homogeneous single crystal rod-like structure with an interplanar spacing of $0.49 \mathrm{~nm}$ (Supplementary, Fig. 3 and Fig. 4). The UV/Vis absorption spectrum (Fig. 1A) and XPS valence band spectrum (Fig. 1B) were measured to confirm the position of valence band (VB) and conduction band (CB) of $\alpha-\mathrm{MnO}_{2}$. The bandgap of $\alpha-\mathrm{MnO}_{2}$ was observed to be $\sim 1.9 \mathrm{eV}$ with a corresponding valence band of $0.78 \mathrm{eV}$ (vs. NHE). The calculated edge positions of $V B_{\alpha-M n O_{2}}$ and $C B_{\alpha-M n O_{2}}$ were $-3.72 \mathrm{eV}$ (vs. vacuum) and $-1.82 \mathrm{eV}$ ( $v s$. 
vacuum), respectively. Fig. 1C shows the schematic diagram of the energy level configuration of the positive electrode in an aqueous aluminum-manganese battery, and the morphology of photo positive electrode was shown in Fig. 5A,B (Supplementary). According to electronic transition characteristics, the optoelectronics would be transmitted to the external circuit along the $\alpha-\mathrm{MnO}_{2} / \mathrm{rGO} / \mathrm{CF}$ while suppressing the migration of holes. Therefore, such feature would ensure the effective separation of the generated electron-hole pairs.

Fig. 1D-G shows the designed schemes of the coin-type photo-excited electrochemical cell. Upon illuminating with the visible light, efficient chargeextraction would be induced in the $\mathrm{MnO}_{2}$ positive electrode, which leads to fast AAIBs charge. In order to simulate the natural sunlight $(400-780 \mathrm{~nm})$, an optical filter was introduced to remove the ultraviolet light below wavelength of $400 \mathrm{~nm}$. Meanwhile, a hole with diameter of $8.0 \mathrm{~mm}$ was placed in the center of the as-assembled coin-type cell for light radiation (Fig. 1D). The configuration scheme of the aluminum-manganese battery was displayed in Fig. 1E. When applying light to the photo positive electrodes upon the charging or discharging processes, the visible light could radiate directly onto the surface of the positive electrode through the hole. In light excitation, the photosensitizer firstly absorbs photons ( $\mathrm{E}_{\mathrm{hv}}>\mathrm{E}_{\mathrm{g}}, \mathrm{E}_{\mathrm{g}}$ : bandgap) to generate electron-hole pairs $^{14}$. Upon absorption of a photon, the electron could be excited from the semiconductor valence band to the conduction band, leaving a hole in the original position. ${ }^{15}$ In order to prevent the non-radiative recombination of electron-hole pairs, the reduced graphene oxide ( $\mathrm{rGO}$ ) was employed as an electron transport layer to 
conduct separated electrons. Compared to a single electrochemical process (Fig. 1F), the photoelectrochemical coupling process would lead to generate optoelectronics to flow out into the external circuit, thereby achieving fast charging and significantly enhanced rate performance (Fig. 1G). Due to the direct radiation of visible light, a photo-oxidation behavior in the photoelectrochemical coupling process could effectively promote the conversion of soluble $\mathrm{Mn}^{2+}$, resulting in high utilization of active materials (part 2.4).

To substantially alleviate the overpotential at the pristine Al negative electrode, a $\mathrm{Zn} / \mathrm{Al}$ alloy negative electrode was also prepared. In the typical process ${ }^{7,16}$, the zinc foil was directly employed as the substrate via an alloying process during the charging stage (Supplementary, Fig. 6A,B). XRD and XPS spectra were performed to confirm the asprepared Zn/Al alloy electrodes (Supplementary, Fig. 7). Moreover, the morphology and element distribution of $\mathrm{Zn} / \mathrm{Al}$ negative electrode was characterized by SEM and EDS (Supplementary, Fig. 8). To verify the electrochemical plating/stripping behavior of $\mathrm{Al}$ in the $\mathrm{Al}(\mathrm{OTF})_{3}$ aqueous electrolyte (current density of $0.2 \mathrm{~mA} \mathrm{~cm}{ }^{-2}$ ), the symmetric cells were assembled using Al, TAl (abbreviations of modified Al foil by acidic ionic liquid), and $\mathrm{Zn} / \mathrm{Al}$ alloy. The $\mathrm{Zn} / \mathrm{Al}-\mathrm{Zn} / \mathrm{Al}$ symmetric cell exhibited the lowest overpotential of $\sim 50 \mathrm{mV}$, and the voltage profiles were also smooth and remained stable over $120 \mathrm{~h}$ (Supplementary, Fig. 9A,B). Overall, utilization of alloying negative electrode is expected to massively enhance the efficiency of $\mathrm{Al}$ plating/stripping and electrochemical performance of AAIBs.

Electrochemical performance. Fig. 2 exhibits the electrochemical performance of the 
as-assembled aqueous aluminum-manganese battery based on $\mathrm{Zn} / \mathrm{Al}$ alloy negative electrode under different conditions (illumination and dark environment). At a scanning rate of $1.0 \mathrm{mV} \mathrm{s}^{-1}$ within the voltage range of $0.5-2.0 \mathrm{~V}$, representative cyclic voltammetry (CV) curves of the batteries exhibit two pairs of oxidation/reduction peaks at $1.45 / 1.4 \mathrm{~V}$ (weak peaks) and 1.9/1.7 V (pronounced peaks), which is attributed to the $\mathrm{Al}^{3+}$ de-intercalation/intercalation process (Fig. 2A). Compared with the battery under dark conditions, the battery upon illumination exhibited a lower polarization voltage with an increased CV area (increment of 56.4\%). The CVs at different scan rates (1.0 to $4.0 \mathrm{mV} \mathrm{s}^{-1}$ ) were also performed and each calculated redox peak current was linear with the square root of the scanning rate (Supplementary, Fig. 10), suggesting that the photo-electrochemical battery was mainly linked with the diffusion control reaction.

Fig. 11 (Supplementary) shows the electrochemical evolution of manganese valence during charging and discharging process. As expected, the high-valence manganese $\left(\mathrm{Mn}^{4+}\right)$ was gradually decreased with the reduction process. In contrast, a reversible evolution was discovered in the oxidation process, which was consistent with the previous literature ${ }^{8}$. In Fig. $2 \mathrm{~B}$, the AAIBs in the dark environment delivered initial discharge capacities of 376.4 and $286.0 \mathrm{~mA} \mathrm{~h} \mathrm{~g}^{-1}$ at 100 and $300 \mathrm{~mA} \mathrm{~g}^{-1}$, respectively. After 100 and 200 cycles, the discharge capacities were maintained at 305.0 and 205.4 $\mathrm{mA} \mathrm{hg}{ }^{-1}$, respectively. Compared with the previously reported $\alpha-\mathrm{MnO}_{2}$ positive electrode, the significantly enhanced capacity and stability is mainly attributed to the reversible plating/stripping of $\mathrm{Al}$ and lowered overpotential on the alloying negative electrode. In the dark environment and under illumination condition, the corresponding 
rate performance of the cell was separately evaluated at various rates from 300 to 1000 $\mathrm{mAg}^{-1}$, as shown in Fig. 2C. In terms of dark environment, the initial discharge capacity $249.5 \mathrm{~mA} \mathrm{~h} \mathrm{~g}^{-1}$ (current density of $300 \mathrm{~mA} \mathrm{~g}^{-1}$ ) was found to decrease to $46 \mathrm{~mA} \mathrm{~h} \mathrm{~g}^{-1}$ (current density of $1000 \mathrm{~mA} \mathrm{~g}^{-1}$ ). Under illuminated conditions, on the contrary, the discharge capacities were significantly increased $\left(203.4 \mathrm{~mA} \mathrm{~h} \mathrm{~g}^{-1}\right)$ even at current density of $1000 \mathrm{~mA} \mathrm{~g}^{-1}$ due to the highly efficient photoelectrochemical coupling process. Interestingly, the corresponding charge/discharge curves at different current densities ((Supplementary, Fig. 12) and (Fig. 2D,E)) exhibited prominent potential platforms and considerable capacity increase. Noticeably, the discharge capacity reached $531 \mathrm{~mA} \mathrm{~h} \mathrm{~g}^{-1}$ (100 $\mathrm{mA} \mathrm{g}^{-1}$ current density) under illumination in comparison with the value $\sim 376.4 \mathrm{~mA} \mathrm{~h} \mathrm{~g}^{-1}$ achieved in the dark environment, which corresponds to a substantial capacity increment of $41.3 \%$ (Fig. 2D). Due to the existence of photooxidation behavior under light, the significant enhancement of discharge capacity can be understood as the coexistence of photo-charging and electrochemical reduction. Compared with other AAIBs systems in the previous studies (Table 1), the photoelectrochemical cells under illumination conditions here presented pronounced enhancement in the specific discharge capacity with outstanding rate performance.

In order to study the dynamic behaviors of AAIBs in the dark environment and under light illumination, the electrochemical impedance spectroscopy (EIS, $10 \mathrm{mV}$ bias voltage) was also acquired (Fig. 2F). Apparently, the total charge transfer impedance ( $\left.\mathrm{R}_{\mathrm{ct}}\right)$ was $200 \Omega$ in the dark environment, while it decreased to $109 \Omega$ under light radiation. Such decreased impedance could be attributed to the accumulation of light- 
excited holes in the positive electrode. A similar phenomenon was also observed in the previous studies ${ }^{17-19}$. Fig. $2 \mathrm{G}$ shows the analytical results of distribution of relaxation times (DRT) achieved from EIS. The $\mathrm{R}_{1}, \mathrm{R}_{2}$, and $\mathrm{R}_{3}$ time regions represented the contact resistance, ion diffusion resistance, and electrochemical reaction resistance ${ }^{20}$, respectively. In comparison, a shortened relaxation time in $\mathrm{R}_{2}$ was obtained under light radiation, indicating faster ion diffusion and kinetics in the solid electrode. Subsequently, the dynamics of AAIBs under dark and illuminated conditions was also performed via galvanostatic intermittent titration technique (GITT) technology. Compared with the dark condition, $\mathrm{D}_{\mathrm{Al}^{3+}}$ have been improved under illumination during discharge, while $\mathrm{D}_{\mathrm{Al}^{3+}}$ almost remains the same during charge process (Fig. $2 \mathrm{H}$ and Supplementary, Fig. 13).

To demonstrate the contribution of photo-regulation in photoelectrochemical coupling process, the photo-conversion efficiency was calculated to be $1.2 \%$ according to the experimental parameters. (See supplementary, Fig. 13 for specific calculation process) Much enhanced performance was mainly attributed to the high photoconversion efficiency. The detailed comparison of the photoelectrochemical coupling systems based on other similar studies was also summarized in Fig. 2I. Obviously, such photoelectrochemically cell exhibited a distinguished discharge capacity and increased rate at different current densities. In addition, the critical performance metrics, including photo-conversion efficiency, light power density, capacity, discharge voltage, and increase the rate of the capacity, were competitive and even superior to the values in recent studies (Fig. 2J and Table 2). 
Light excitation response. To fully understand the fast charging and enhanced rate performance of the photo-excited aluminum-manganese battery, it is significant to study the carrier dynamics and transport. Fig. 3A-C shows the transient absorption map, spectra, and dynamics of a spin-coated $\alpha-\mathrm{MnO}_{2}$ thin film using $400 \mathrm{~nm}$ pump photons. As shown in Fig. 3A,B, a strong optical excitation absorption signal was captured around $600 \mathrm{~nm}$, suggesting the generation of electron-hole pairs in the $\alpha-\mathrm{MnO}_{2}$ crystal. Subsequently, the separated photo-excited electrons can transport with matched energy levels, resulting in the fast-charging rates in the photo-electrochemical battery. Generally, the recombination rate of electron-hole pairs is linked with carrier lifetime. Apparently, longer time delay would lead to increased photoinduced electrons. Therefore, the carrier lifetime of both $\alpha-\mathrm{MnO}_{2}$ semiconductor and mixture of $\alpha-\mathrm{MnO}_{2}$ and $\mathrm{rGO}\left(\alpha-\mathrm{MnO}_{2}-\mathrm{rGO}\right)$ was evaluated to understand photo-charge carrier dynamics. Both $\alpha-\mathrm{MnO}_{2}$ and $\alpha-\mathrm{MnO}_{2}-\mathrm{rGO}$ exhibited only slight signal decay, indicating that their carrier lifetime is much greater than $7.5 \mathrm{~ns}$ (Fig. 3C). Additionally, the $\alpha-\mathrm{MnO}_{2}-\mathrm{rGO}$ presented greater charge carrier dynamics, and it is attributed to the electron transport layer (rGO) that plays a positive effect role in charge separation and transfer. To further confirm the generation and separation process of the photo-induced electrons, the photo-current response upon layer-by-layer transparent photodetector was measured. In the configuration of $\mathrm{FTO} / \mathrm{rGO} / \mathrm{MnO}_{2} / \mathrm{Ag}$ photodetector, $\mathrm{Ag}$ is the positive electrode and FTO layer is the negative electrode, as shown in Fig. 3D. The photocurrent response (vs. open circuit potential, i.e., $0 \mathrm{~V}$ bias voltage) was evaluated via several on/off cycles of the optical radiation, as shown in Fig. 3E. The photocurrent rapidly increased to 
$0.45 \mu \mathrm{A} \mathrm{cm} \mathrm{cm}^{-2}$ when the light was turned on. Then, the photocurrent decreased to $0 \mu \mathrm{A}$ $\mathrm{cm}^{-2}$ when the light was turned off, suggesting good cycling stability and highefficiency extraction of photogenerated electrons. When a $10.0 \mathrm{mV}$ bias voltage was applied to the electrode, the photocurrent was increased to $0.55 \mu \mathrm{A} \mathrm{cm}{ }^{-2}$ (Fig. 3F), which indicates that the bias voltage could effectively promote the separation and transportation of photogenerated electron-hole pairs. To further study the separation and transfer of photoexcited electrons in the static electrochemical system, the photocurrent response of AAIBs (initial, fully discharged to $0.5 \mathrm{~V}$ (D-0.5 V), and fully charged to 2.0 $\mathrm{V}(\mathrm{C}-2.0 \mathrm{~V})$ ) was evaluated under on/off state of the visible light illumination (Fig. 3G). When the light was on, a photocurrent $\sim 44.5 \mu \mathrm{A} \mathrm{cm}^{-2}$ was obtained in the initial AAIBs. Once the light switched off, it decreased to zero. The photocurrent of AAIBs at fully charged and discharged states exhibited almost the equal intensity (D-0.5 V: $42.5 \mu \mathrm{A}$ $\mathrm{cm}^{-2}, \mathrm{C}-2.0: 43.2 \mu \mathrm{A} \mathrm{cm} \mathrm{cm}^{-2}$ ) under on/off state of the visible light illumination. The obtained photocurrent was measured by chronoamperometry at $0 \mathrm{~V}$ bias voltage, indicating that the photocurrent was generated by the effective extraction of photoexcited electrons. The corresponding several consecutive cycles of photocurrent-time curves and photocurrent values were described in Fig. 14 (Supplementary) and Fig. 3H, respectively. Apparently, the photocells at states of pre-test, D-0.5 V and C-2.0 V all exhibited a stable and almost the equal photocurrent. In particular, the observed higher response current of the first cycle in D- $0.5 \mathrm{~V}$ and $\mathrm{C}-2.0 \mathrm{~V}$ might be caused by the initial unsteady state of the cells. Compared with $\mathrm{FTO} / \mathrm{rGO} / \mathrm{MnO}_{2} / \mathrm{Ag}$ photodetector, the response current was significantly enhanced, which is attributed to the photo-oxidation 
behavior of the photo positive electrode, i.e., the photo-charging process in the chemical cells. Precisely, this phenomenon is due to the rapid separation and transmission of the photoinduced electrons in a high response current, and thus achieving fast charging and high-rate capabilities. Additionally, compared with solid state photodetectors, the delay of the photocurrent response was observed in the chemical cells, which might attribute to the adsorption of liquid electrolyte.

Photoelectrochemical coupling mechanism. According to the light excitation response behaviors, a large photocurrent was generated due to the high-efficiency separation and transmission of photoelectrons. Therefore, fast charging and enhanced rate performance of the cells could be achieved during the photoelectrochemical coupling process. Therefore, it is significant to understand the mechanism of the photoelectrochemical coupling process.

Fig. 4A shows the intermittent charge and discharge curves under dark and illumination conditions at a current density of $100 \mathrm{~mA} \mathrm{~g}^{-1}$. In the charge process, the electrode potential will be positively shifted along with the electrochemical oxidation. On the contrary, the electrode potential in the discharge process will be negatively shifted upon the electrochemical reduction process. Upon charge in the dark environment, however, the electrode potential was observed to be negatively shifted by $\sim 20 \mathrm{mV}\left(\Delta \mathrm{V}_{\mathrm{C}}=20 \mathrm{mV}\right.$, Fig. 4B $)$ when the light was applied. Subsequently, the electrode potential continued to shift forward with further illumination. When the illumination was off, this phenomenon was cut-off and the electrode potential was found to shift significantly forward. As expected, the opposite phenomenon was observed during the 
discharging process. A positive shift $\left(\Delta \mathrm{V}_{\mathrm{D}}=100 \mathrm{mV}\right)$ was observed in the electrode potential when the cell was exposed to illumination (Fig. 4A). In the charging and discharging process, the shifted potential would be mainly attributed to the characteristic of n-type semiconductors, it may be associated with the negative charge accumulation in the conduction band under illumination. Meanwhile, the results of a significant abrupt change in the electrode potential also confirm that $\alpha-\mathrm{MnO}_{2}$ is highly sensitive to light in the electrochemical process. Upon illumination, an apparent shift in the potential $\left(\Delta \mathrm{V}_{\mathrm{D}}>\Delta \mathrm{V}_{\mathrm{C}}, \Delta \mathrm{V}=80 \mathrm{mV}\right)$ was exhibited in the charging and discharge processes. To further probe the shift behavior on electrode potential, density functional theory (DFT) calculation was carried out. Fig. 15A (Supplementary) shows the structure model of an $\alpha-\mathrm{MnO}_{2}$ unit. Subsequently, three intercalation models $\left(\mathrm{Al}_{1}\left(\mathrm{MnO}_{2}\right)_{18}, \mathrm{Al}_{2}\left(\mathrm{MnO}_{2}\right)_{18}\right.$, and $\left.\mathrm{Al}_{3}\left(\mathrm{MnO}_{2}\right)_{18}\right)$ were established and optimized to verify the electronic variation of $\alpha-\mathrm{MnO}_{2}$. Fig. 15B-D (Supplementary) shows the corresponding electron density difference and the charge was mainly concentrated near the intercalated $\mathrm{Al}^{3+}$, indicating that the $\mathrm{Al}^{3+}$ could be fixed stably due to the strong interaction with $\mathrm{MnO}_{2}$. The bandgap of the original $\alpha-\mathrm{MnO}_{2}$ was calculated as $\sim 2.1 \mathrm{eV}$ in the electronic band (Supplementary, Fig. 15E), which was approximate to the experimental results $\left(\sim 1.9 \mathrm{eV}\right.$, Fig. 1E). After intercalating $\mathrm{Al}^{3+}$, there was almost no change in the positions of the conduction band and valence band of $\alpha-\mathrm{MnO}_{2}$, which demonstrates that the bandgap of $\mathrm{Al}_{1}\left(\mathrm{MnO}_{2}\right)_{18}, \mathrm{Al}_{2}\left(\mathrm{MnO}_{2}\right)_{18}$, and $\mathrm{Al}_{3}\left(\mathrm{MnO}_{2}\right)_{18}$ was consistent with $\alpha-\mathrm{MnO}_{2}$ (Supplementary, Fig. 15F-H). The corresponding partial density of states (PDOS) (Supplementary, Fig. 15I-L) further confirmed that the 
intercalated $\mathrm{Al}^{3+}$ would introduce hybrid level, and whereby the transition barrier of photo-excited electrons from the valence band to the conduction band could be effectively decreased (Fig. 4C). The results from DFT calculation would be used to clarify the shifting phenomenon in the electrode potential, as mentioned in Fig. 4A. Namely, the introduction of hybrid energy levels significantly promoted the migration of optoelectronics from the valence band to the conduction band during the discharge process, thus leading to a more pronounced significant shift in electrode potential.

In order to determine the chemical changes induced by photoexcitation and clarify the photoelectrochemical coupling mechanism, electron paramagnetic resonance (EPR) was carried out under the conditions with and without applying illumination a photo positive electrode. Fig. 4D shows the EPR spectrum and the corresponding g value distribution is exhibited in Fig. 4E. With or without illumination, six typical characteristic signals were all observed at 300-400 $\mathrm{mT}(\mathrm{g} \approx 2.0)$ (Fig. 4E), which was defined as $\mathrm{Mn}^{2+21}$. In the EPR spectra, however, there is lacked of signal related to $\mathrm{Mn}^{3+}$ due to the integer $\operatorname{spin}^{22}$. Accompanied with illumination, a higher g-value $(g \approx 3.8)$ signal was observed at $100-200 \mathrm{mT}$ (defined as $\mathrm{Mn}^{4+}$ ), which was in sharp contrast to the signal under dark condition ${ }^{23}$. This phenomenon demonstrates that photo-radiation could promote the transformation of low-valence manganese $\left(\mathrm{Mn}^{2+}\right.$, including partial $\left.\mathrm{Mn}^{3+}\right)$ to high-valence manganese $\left(\mathrm{Mn}^{4+}\right)$. Therefore, it is reasonable that the photooxidation behavior existed in the photo positive electrode, which can be described as following reaction equation:

$$
\mathrm{Mn}^{2+} \stackrel{\mathrm{hv}}{\rightarrow}\left[\mathrm{Mn}^{2+}\right]^{*} \stackrel{\mathrm{hv}}{\rightarrow} \mathrm{Mn}^{4+}\left(\mathrm{h}^{+}\right)+\left(\mathrm{e}^{-}\right)
$$


Under the dark condition, the stable operation of AAIBs could be achieved in a single electrochemical process. Upon illumination, two steps, including photochemical and electrochemical (defined as a photoelectrochemical coupling process), would participate in the electrode process. In addition to the electrochemical process, the photo-oxidation process would also release electrons (photoinduced electrons) and flowed to the negative electrode through the external circuit (Fig. 4F). Meanwhile, the remaining holes would be neutralized by the electrons generated in the process of $\mathrm{Al}^{3+}$ deintercalation. In the discharge process, the holes were consumed by electrons coming from an external circuit, while the photoinduced electrons would be taken along with the intercalation of $\mathrm{Al}^{3+}$.

The dissolution of $\mathrm{Mn}^{2+}$ was an undesirable chemical behavior in the electrochemical process. Interestingly, such behavior could be suppressed by the photooxidation process. Fig. 4G shows the Raman spectroscopy of the electrolytes after cycles, and the Mn-O (580 $\mathrm{cm}^{-1}$, in the range of 550-620 $\left.\mathrm{cm}^{-1}\right)$ chemical bond was observed. To further verify the inhibitory effect of photo-oxidation behavior on soluble $\mathrm{Mn}^{2+}$, inductively coupled plasma mass spectrometry was carried out to track the concentration of $\mathrm{Mn}^{2+}$ in the electrolyte after different cycles (Fig. 4H). In comparison with dark conditions, the manganese concentration in the electrolyte had been effectively suppressed under the illuminated condition, which indicates that the electrochemically reduced soluble $\mathrm{Mn}^{2+}$ was subsequently conversed to $\mathrm{Mn}^{4+}$ by the photo-oxidation process. As a result, unnecessary loss of $\mathrm{Mn}^{2+}$ could be effectively alleviated. Fig. 4I shows the charging (with illumination) and discharging (with and 
without illumination) curves at a current density of $100 \mathrm{~mA} \mathrm{~g}^{-1}$. Upon photoelectrochemical coupling process, a longer discharge time was obtained, indicating that more $\mathrm{Al}^{3+}$ was intercalated and greater energy was released. Meanwhile, the charging experiments under light and dark conditions (current density of $100 \mathrm{~mA} \mathrm{~g}^{-}$ $\left.{ }^{1}\right)$ followed by dark galvanostatic discharge cycles at different current densities (100, 200, and $300 \mathrm{~mA} \mathrm{~g}^{-1}$ ) were also performed (Fig. 4J). In dark discharge, the light-charged cell exhibited a longer discharge time, which fully demonstrated the advantages of photoelectrochemical coupling process.

\section{Discussion}

In this study, a prototype of a stable photoelectrochemical coupling system was designed by a simple photo-regulation strategy. Porous carbon fiber paper and a small amount of rGO were employed as current collectors and ETL, respectively. Thus, large area of light radiation and efficient separation and transmission of photoelectrons could be achieved. Compared with those strategies based on physical and chemical approaches, the photo-regulation strategy using $\alpha-\mathrm{MnO}_{2}$ positive electrode has presented great competitive.

Nevertheless, there is plenty of room for further enhancing the photoelectrochemical coupling efficiency via rational design of photo positive electrode, such as increasing light radiation area or decreasing the recombination of electron-hole pairs. Therefore, development of more efficient energy level matching of photo positive electrode along with a highly optically transparent current collector should be the critical challenges in the future. To reach the goals, the current collectors including FTO, 
ITO, and porous carbon fibers, would be further explored. The design strategies here also open an ideal route that allows for extending into other photo-chemical or photoelectrochemical coupling systems and processes.

For substantially overcoming the issues of low energy density and slow charge rate in the aqueous aluminum-manganese batteries, a novel photo-regulation strategy has been proposed and a stable photoelectrochemical coupling system has been established. Based on the electrochemical process of $\alpha-\mathrm{MnO}_{2}$, a photoelectrochemical coupling approach was investigated to achieve fast charging and enhance rate performance. Meanwhile, the photo-oxidation behavior was also observed on the photo positive electrode, and the loss of soluble $\mathrm{Mn}^{2+}$ was effectively alleviated as expected. Because of the stable and high-efficiency photoelectrochemical coupling process, a photoelectric conversion efficiency $1.2 \%$ has been obtained. Nevertheless, great efforts could be still paid to optimize the photoelectrochemical coupling systems, such as development of a more stable anode and electrolyte. It is believed that rational manipulation approach can be developed to promote the practical applications in various advanced photoelectrochemical engineering and devices.

\section{Methods}

Synthesis of $\alpha-\mathrm{MnO}_{2}$ nanorod. Single crystal $\alpha-\mathrm{MnO}_{2}$ nanotubes were prepared by a simple hydrothermal approach. ${ }^{8}$ In a typical preparation, $5 \mathrm{mM} \mathrm{KMnO}_{4}$ was firstly dissolved into the solution of $24 \mathrm{~mL}$ of $1.0 \mathrm{M} \mathrm{HCl}$ with continuous string to obtain a homogeneous solution. Then, the $46 \mathrm{~mL}$ of distilled water was slowly introduced abovementioned solution. After stiring at room temperature for $30 \mathrm{~min}$, the solution 
was transferred into a Teflon-lined stainless-steel autoclave. The reactor was stayed at $140^{\circ} \mathrm{C}$ and reacted for 18 hours. After cooling to room temperature, the $\alpha-\mathrm{MnO}_{2}$ was separated using a centrifuge from the suspension solution, followed by cleaning with deionized water and alcohol several times and drying at $60{ }^{\circ} \mathrm{C}$ overnight.

Preparation of aqueous electrolytes. The $2 \mathrm{M} \mathrm{Al}\left(\mathrm{CF}_{3} \mathrm{SO}_{3}\right)_{3}$ aqueous electrolyte was prepared in an external environment. Firstly, the $9.48 \mathrm{~g}$ of $\mathrm{Al}\left(\mathrm{CF}_{3} \mathrm{SO}_{3}\right)_{3}$ was slowly added into $10 \mathrm{~mL}$ deionized water with continuous stirring. Subsequently, the electrolyte was maintained at room temperature for $12 \mathrm{~h}$ before use.

Preparation of working electrodes. The photo positive electrodes were fabricated by a blade coating approach. In detail, the active materials $\left(\alpha-\mathrm{MnO}_{2}\right)$, conductive agent (acetylene black: $\mathrm{rGO}=8: 2$ ), and binder (PVDF) with a weight ratio of 8:1:1 were mixed with NMP (N-Methyl-2-pyrrolidone) to obtain the homogeneous slurry. After that, the above slurry was coated on carbon fiber paper (CF) (Toray H090) and dried at $65^{\circ} \mathrm{C}$ in an oven overnight. The mass loading of active material was $0.8-1.0 \mathrm{mg} \mathrm{cm}^{-2}$. The photodetector $\left(\mathrm{FTO} / \mathrm{rGO} / \mathrm{MnO}_{2} / \mathrm{Ag}\right)$, aiming to detect the optical response behavior of $\mathrm{MnO}_{2}$, was prepared by a simple spin coating. In detail, the rGO solution and $\mathrm{MnO}_{2}$ (dispersed in NMP) were layer-by-layer spin coated on the fluorine-doped tin oxide (FTO) coated glass substrate. Finally, the Ag paste contacts were deposited on the top side of the light-absorbing layer, followed by drying at $65^{\circ} \mathrm{C}$.

Assembling of photoelectrochemical batteries. The electrochemical performance of photo-electrochemical batteries was measured by CR2032 coin cell. On the positive 
electrode side, an $8.0 \mathrm{~mm}$ hole was machined on the case of CR2032 coin cell for illumination. The zinc foil was direct as the negative electrode, and the separator (Whatman glass, GF/A) was placed on the top side of the negative electrode. Subsequently, the $2 \mathrm{M} \mathrm{Al}\left(\mathrm{CF}_{3} \mathrm{SO}_{3}\right)_{3}$ aqueous electrolyte was added, followed by the photo positive electrode was placed on top of the separator. After that, a stainless-steel disk spacer with spring and positive electrode case were orderly assembled. Finally, the cell was sealed with an optical glass by epoxy glue.

Electrochemical measurements of photo batteries. The electrochemical performance of the batteries in the conditions of dark and illuminated was carried out by galvanostatic charge/discharge testing system (Neware BTS-53) at room temperature. In this work, the light of wavelength and intensity were $400-780 \mathrm{~nm}$ visible light and $100 \mathrm{~mW} \mathrm{~cm}^{-2}$ (300 W Xenon lamp, PerfectLight, Co., Ltd, Beijing, China). Cyclic voltammetry $(\mathrm{CV})$ was performed with a $\mathrm{CHI} 660 \mathrm{E}$ electrochemical workstation. The photo-response current was taken by potentiostatic method (Solartorn, ELECTROCHEMICAL INTERFACE, SI 1287) under alternating dark and illuminated conditions in the absence of an external bias. The GITT curves were performed by alternating between 30 minutes of applied current and 10 minutes of rest at a current density of $0.1 \mathrm{~mA} \mathrm{~cm}^{-2}$.

Material Characterization. The crystal structures and elemental valences of the raw materials and positive electrodes after charge/discharge were characterized by XRD (Rigaku, D/max-RB, Cu Ka wave, $1=1.5418 \AA$ ) and XPS (a Kratos AXISUITRA-DLD 
spectrometer using an Al Ka source). The morphological and elemental analysis of $\mathrm{MnO}_{2}$ samples were characterized by SEM and TEM (JEOL, JEM-2010). The optical properties of the samples were characterized by using UV-VIS-NIR Spectrometer (PE lambda 750)

TA and EPR measurements. In order to investigate the optical kinetics behaviors of $\alpha-\mathrm{MnO}_{2}$, the transient absorption (TA) measurements were performed using a HELIOS spectrometer. An amplified Ti: sapphire laser with a chopper actual frequency of $75 \mathrm{~Hz}$ was employed to obtain $400 \mathrm{~nm}$ pump pulses. Moreover, the $\mathrm{Yb}$ lasers were used to achieve the $460-800 \mathrm{~nm}$ probe spectral range. For TA measurements, two film samples were fabricated by a simple spin-coating method. Specifically, homogeneous $\alpha-\mathrm{MnO}_{2}$ and $\alpha-\mathrm{MnO}_{2}$-rGO solutions (NMP as solvent) were coated on the quartz substrate and dried at $100{ }^{\circ} \mathrm{C}$ for $12 \mathrm{~h}$. The EPR measurements in dark and light conditions were carried out employing Bruker A300 spectrometer (EMXmicro-6/1/P/L, Karlsruhe, Germany). The sample was a photo positive electrode that had been discharged to 0.5 $\mathrm{V}$, to obtain a large amount of low-valence manganese. Light illumination was provided by a $300 \mathrm{~W}$ Xenon lamp under a low temperature of $70 \mathrm{~K}$.

\section{Data availability}

Source data are provided with this paper. The other data that support the findings of this study are available from the corresponding author upon request.

\section{References}

1 Tu, J. et al. Nonaqueous Rechargeable Aluminum Batteries: Progresses, 
Challenges, and Perspectives. Chem. Rev. 121, 4903-4961 (2021).

2 Jiao, $\mathrm{H}$. et al. $\mathrm{Cu}-\mathrm{Al}$ composite as the negative electrode for long-life Al-ion batteries. J. Electrochem. Soc. 166, A3539 (2019).

3 Jiao, H. et al. Al homogeneous deposition induced by $\mathrm{N}$-containing functional groups for enhanced cycling stability of Al-ion battery negative electrode. Nano Res. 14, 646-653 (2021).

4 Cai, Y. et al. Bronze-type vanadium dioxide holey nanobelts as high performing cathode material for aqueous aluminium-ion batteries. J. Mater. Chem. A 8 , 12716-12722 (2020).

$5 \mathrm{Wu}$, X. et al. Reversible aluminum ion storage mechanism in Ti-deficient rutile titanium dioxide anode for aqueous aluminum-ion batteries. Energy Storage Mater. 37, 619-627 (2021).

6 Chen, J. et al. Rechargeable aqueous aluminum organic batteries. Angew. Chem. 133, 5858-5863 (2021)

7 Yan, C. et al. Architecting a stable high-energy aqueous al-ion battery. J. Am. Chem. Soc. 142, 15295-15304 (2020).

8 Zhao, Q. et al. Solid electrolyte interphases for high-energy aqueous aluminum electrochemical cells. Sci. Adv. 4, eaau8131 (2018).

$9 \mathrm{Wu}, \mathrm{C}$. et al. Electrochemically activated spinel manganese oxide for rechargeable aqueous aluminum battery. Nat. Commun. 10, 1-10 (2019).

10 He, S. et al. A high-energy aqueous aluminum-manganese battery. Adv. Funct. Mater. 29, 1905228 (2019). 
11 Wang, P. et al. A flexible aqueous Al ion rechargeable full battery. Chem. Eng. J. 373, 580-586 (2019).

12 Cockayne, E. \& Li, L. First-principles DFT+ U studies of the atomic, electronic, and magnetic structure of $\alpha-\mathrm{MnO}_{2}$ (cryptomelane). Chem. Phys. Lett. 544, 5358 (2012).

13 Ramesh, M., Nagaraja, H., Rao, M. P., Anandan, S. \& Huang, N. Fabrication, characterization and catalytic activity of $\alpha-\mathrm{MnO}_{2}$ nanowires for dye degradation of reactive black 5. Mater. Lett. 172, 85-89 (2016).

14 Mazzio, K. A. \& Luscombe, C. K. The future of organic photovoltaics. Chem. Soc. Rev. 44, 78-90 (2014).

15 He, J., Lindström, H., Hagfeldt, A. \& Lindquist, S.-E. Dye-sensitized nanostructured p-type nickel oxide film as a photocathode for a solar cell. $J$. Phys. Chem. B 103, 8940-8943 (1999).

16 Wang, S.-B. et al. Lamella-nanostructured eutectic zinc-aluminum alloys as reversible and dendrite-free anodes for aqueous rechargeable batteries. Nat. Commun. 11, 1-9 (2020).

17 Boruah, B. D. et al. Photo-rechargeable zinc-ion batteries. Energy Environ. Sci. 13, 2414-2421 (2020).

18 Deka Boruah, B. et al. Vanadium dioxide cathodes for high-rate photorechargeable zinc-ion batteries. Adv. Energy Mater. 11, 2100115 (2021).

19 Song, H. et al. Solar-driven all-solid-state lithium-air batteries operating at extreme low temperatures. Energy Environ. Sci. 13, 1205-1211 (2020). 
20 Zhou, X., Huang, J., Pan, Z. \& Ouyang, M. Impedance characterization of lithium-ion batteries aging under high-temperature cycling: Importance of electrolyte-phase diffusion. J. Power Sources 426, 216-222 (2019).

21 Murase, N. et al. Fluorescence and EPR characteristics of $\mathrm{Mn}^{2+}$-doped $\mathrm{ZnS}$ nanocrystals prepared by aqueous colloidal method. J. Phys. Chem. B 103, 754760 (1999).

22 Geng, F. et al. Anionic redox reactions and structural degradation in a cationdisordered rock-salt $\mathrm{Li}_{1.2} \mathrm{Ti}_{0.4} \mathrm{Mn}_{0.4} \mathrm{O}_{2}$ cathode material revealed by solid-state NMR and EPR. J. Mater. Chem. A 8, 16515-16526 (2020).

23 Chen, L. et al. The effect of electron cloud expansion on the red luminescence of $\mathrm{Sr}_{4} \mathrm{Al}_{14} \mathrm{O}_{25}: \mathrm{Mn}^{4+}$ revealed by calculation of the racah parameters. J. Alloys Compd. 613, 312-316 (2014).

\section{Acknowledgements}

This work was supported by National Natural Science Foundation of China (Nos. 51725401, 51874019 and 52074036), Fundamental Research Funds for the Central Universities (No. FRF-TP-17-002C2), Technology Innovation Program of Beijing Institute of Technology (No. 2019CX01021) and BIT Teli Young Fellow.

\section{Author contributions}

X. F. Zhang, S. Q. Jiao, M. Y. Wang, and W. L. Song conceived and designed the experiments. X. F. Zhang performed the experiments. X. F. Zhang and H. D. Jiao analyzed and interpreted the data, X. F. Zhang and W. L. Song co-wrote the paper. S. 
Q. Jiao and M. Y. Wang supervised the project.

\section{Competing interests}

The authors declare no competing interests 

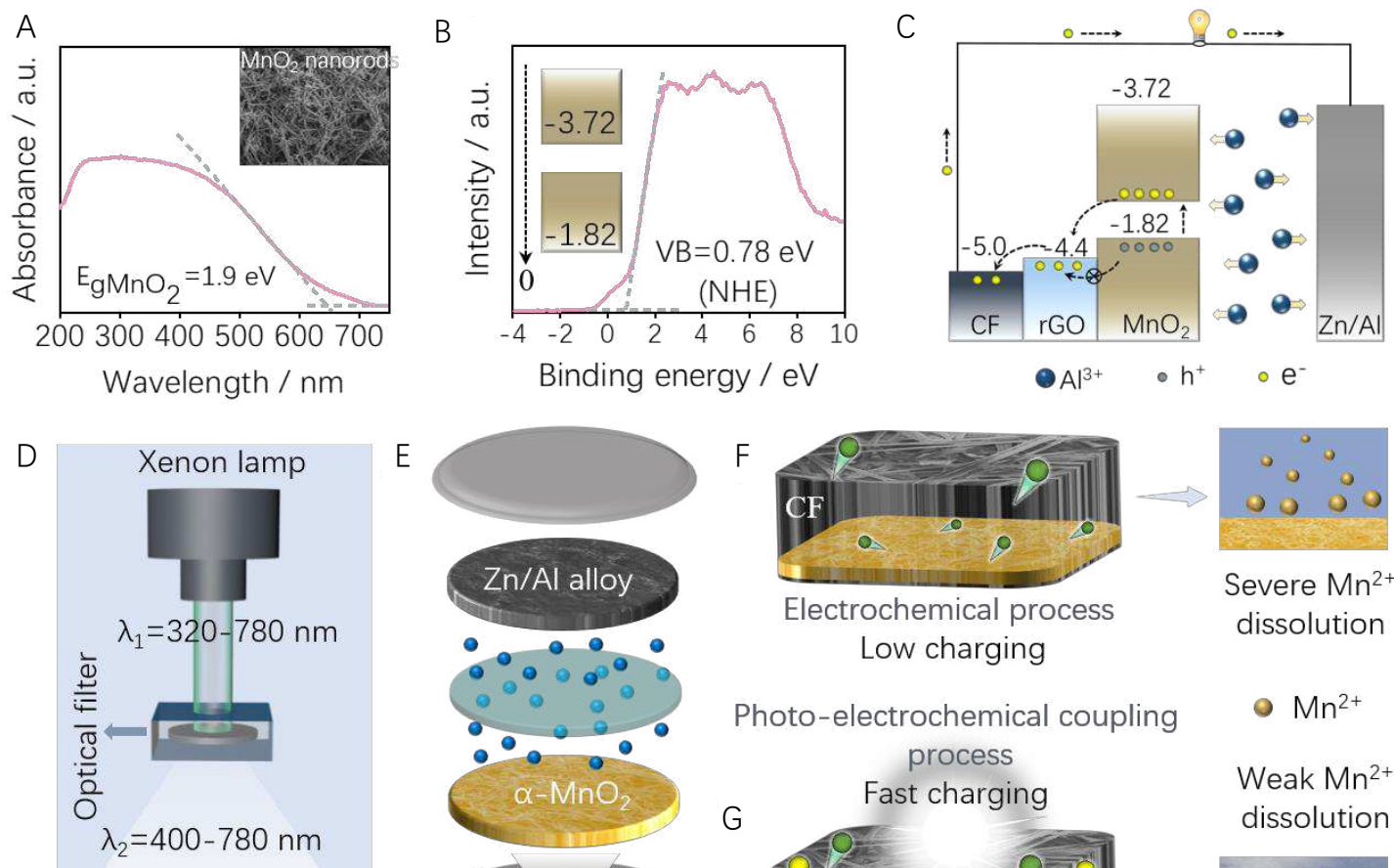
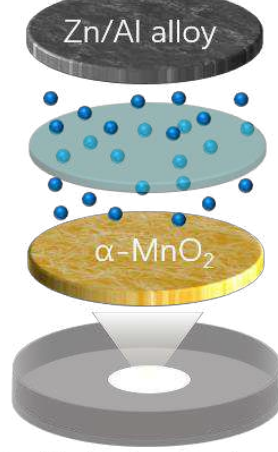

Optoelectronics
Photo-electrochemical coupling $\odot \mathrm{Mn}^{2+}$

G Fast charging

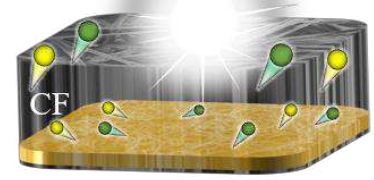

Electrochemical electronics
$\mathrm{Mn}^{2+}$

Weak $\mathrm{Mn}^{2+}$ dissolution

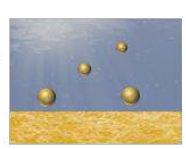

Fig. 1 The schematic diagram of photo-actuated aqueous aluminum manganese

cells. (A) Light radiation coin cell and (B) corresponding configuration diagram. (C,D)

The electrochemical process and photoelectrochemical coupling process of photo positive electrode. (E) UV-Vis spectrum of $\alpha-\mathrm{MnO}_{2}$ nanorods with a bandgap of $\sim 1.9$ eV. (F) The XPS valence band spectrum of $\alpha-\mathrm{MnO}_{2}$ nanorods. (G) The separation process of electron-hole pairs based on energy level matching. 

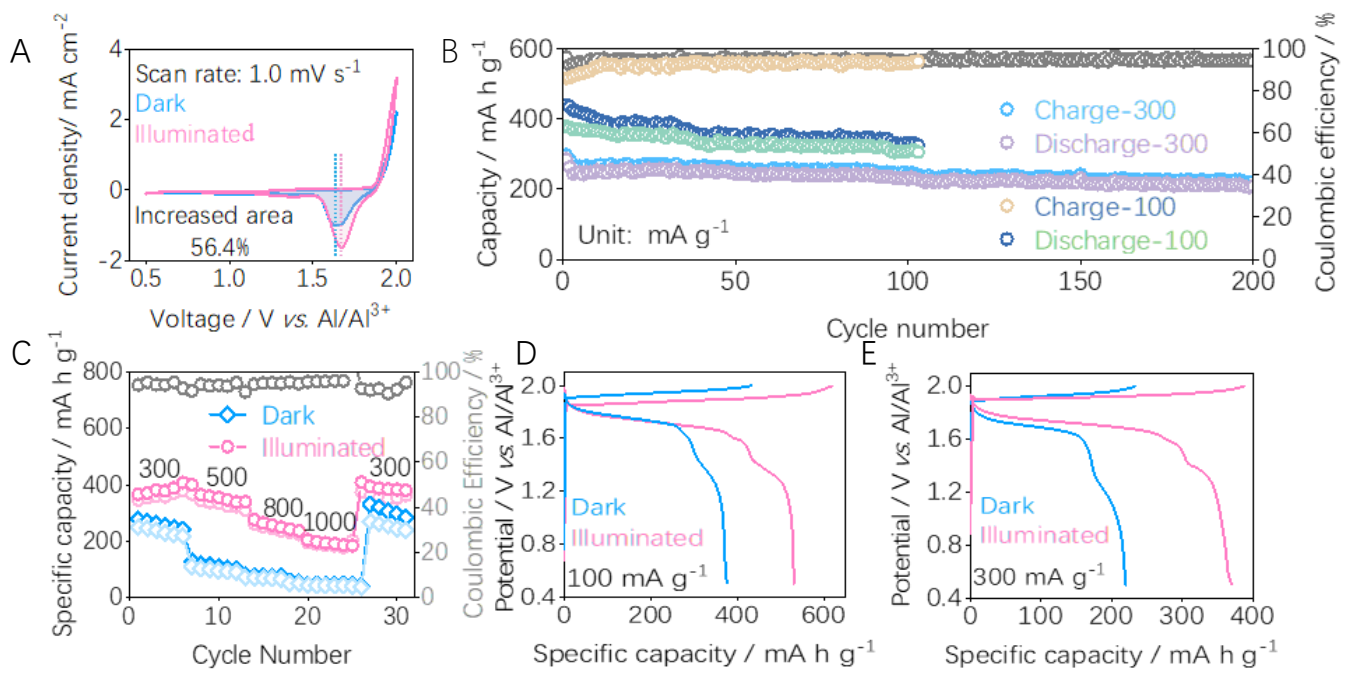

F
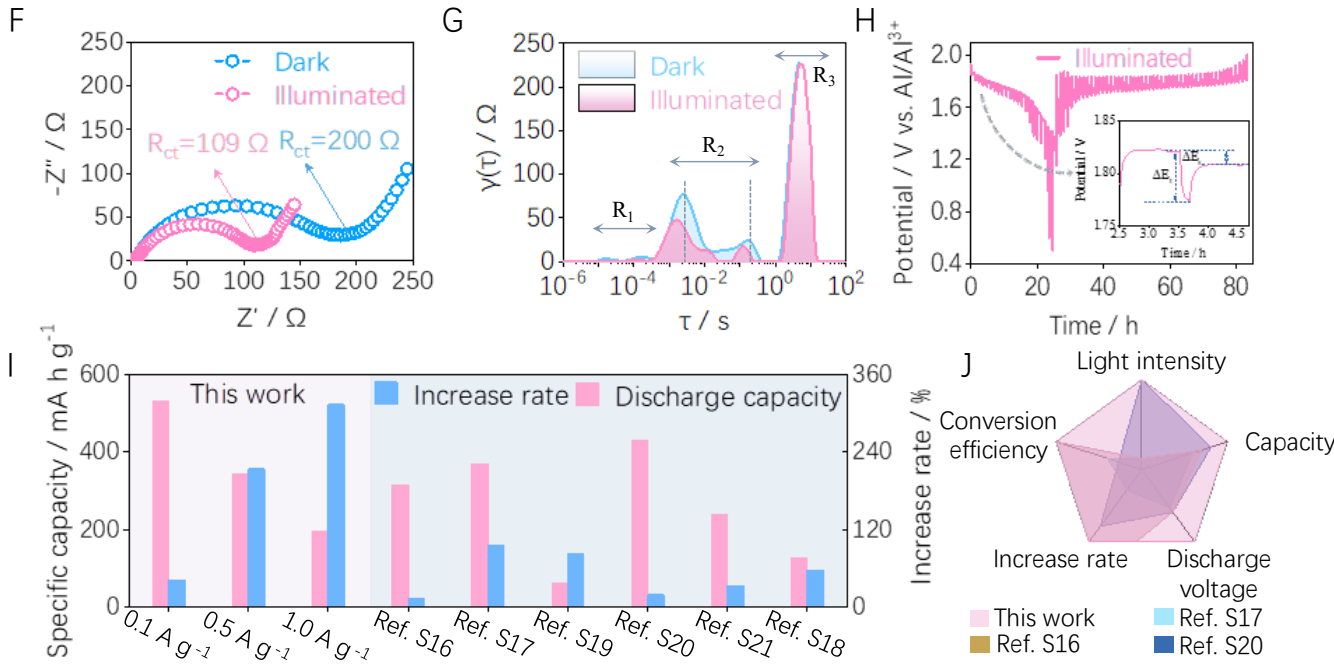

Fig. 2 The electrochemical performance of photo-actuated aqueous aluminum manganese cells under dark and illumined conditions. (A) The CV curves at $1.0 \mathrm{mV}$ $\mathrm{s}^{-1}$ in dark and illuminated conditions. (B) Cycling performance at current densities of 100 and $300 \mathrm{~mA} \mathrm{~g}^{-1}$ without illumination. (C) the rate performance in the "light-off" and "light-on" states. (D, E) Galvanostatic charge/discharge curves carried out with/without illumination at 100 and $300 \mathrm{~mA} \mathrm{~g}^{-1}$, respectively. (F) The capacity and corresponding increase rate comparison of this work at different current densities $\left(\mathrm{A} \mathrm{g}^{-}\right.$ $\left.{ }^{1}\right)$ with the other works. (G) Compare important baseline performance metrics such as conversion efficiency, light intensity, capacity, discharge voltage, and increase rate with other works. (H) Discharge and charge curves of the GITT measurement under 
illuminated conditions for the photo-actuated aluminum-manganese cells. The inset is a single current pulse with a specific setting parameter of 10 min pulse time, $100 \mathrm{~mA} \mathrm{~g}^{-}$ ${ }^{1}$ current pulse, and 30 min relaxation time. (I) Nyquist plots of the EIS spectra under dark and illuminated conditions at $0 \mathrm{~V}$ bias voltage. (J) The DRT results of the corresponding EIS spectra. 

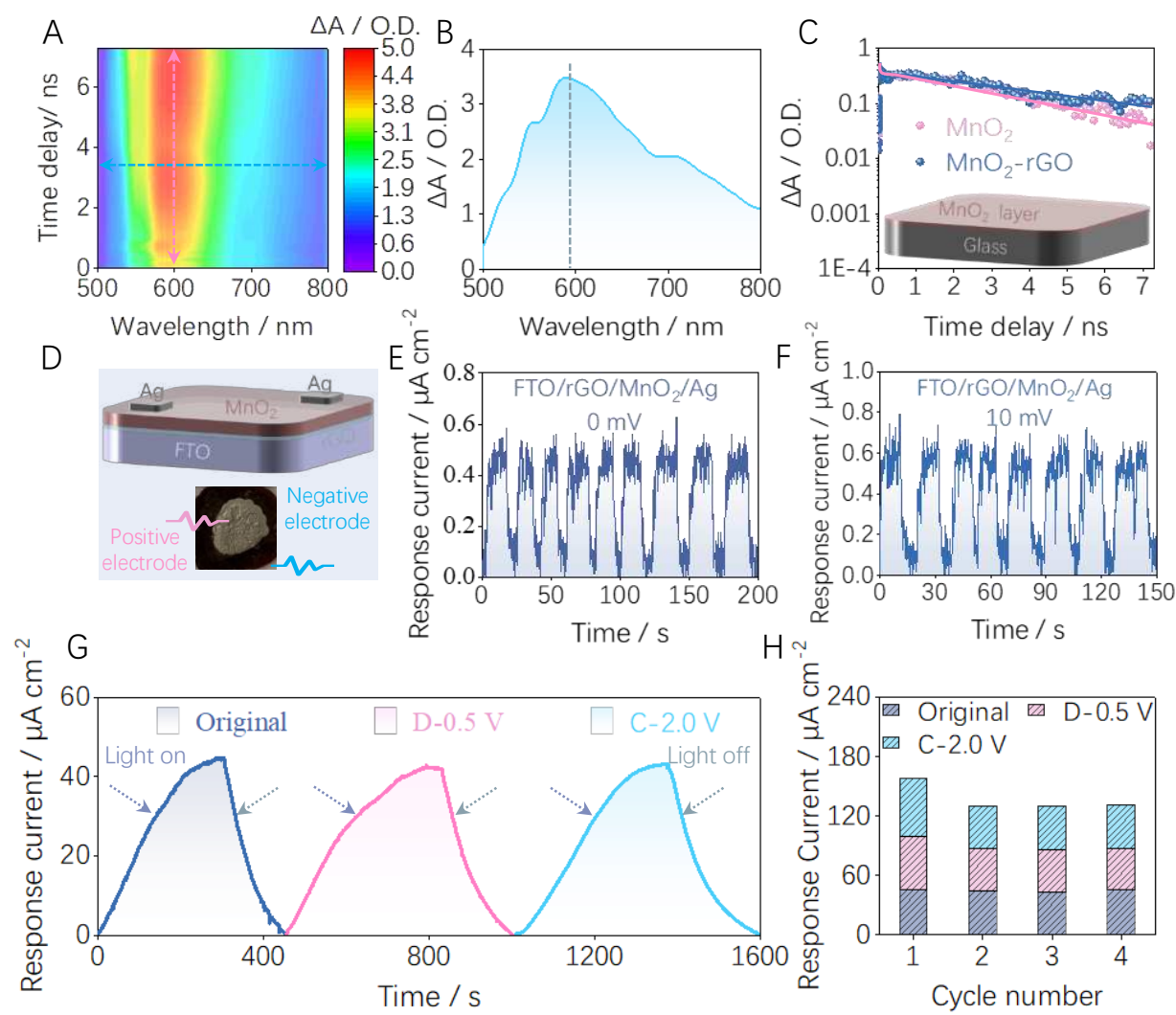

Fig. 3 Light excitation response of layer-by-layer transparent photodetector and

photo positive electrode. (A) TA map and (B) spectrum. (C) TA dynamics of $\alpha-\mathrm{MnO}_{2}$ and $\alpha-\mathrm{MnO}_{2}-\mathrm{rGO}$. Inset shows the schematic diagram of thin-film samples. (D) The digital photo of layer-by-layer transparent photodetector (down) and corresponding schematic diagram (up). (E,F) Photocurrent response of layer-by-layer transparent photodetector at $0 \mathrm{~V}$ and $10 \mathrm{mV}$ bias voltage, respectively. (G) Photocurrent response of photo positive electrode of aluminum-manganese cells at different states, such as original, fully discharged to $0.5 \mathrm{~V}$ and fully charged to $2.0 \mathrm{~V}$. (H) The corresponding value of photocurrent at different cycles. 

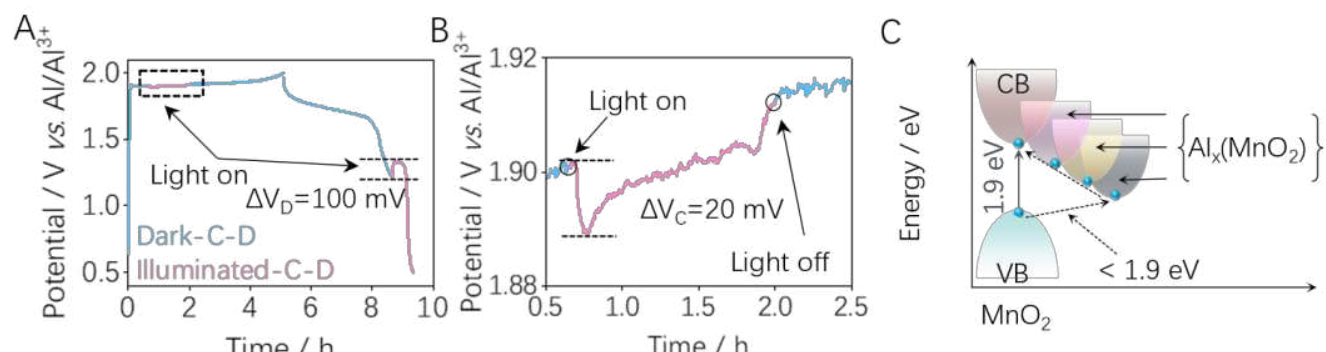

D
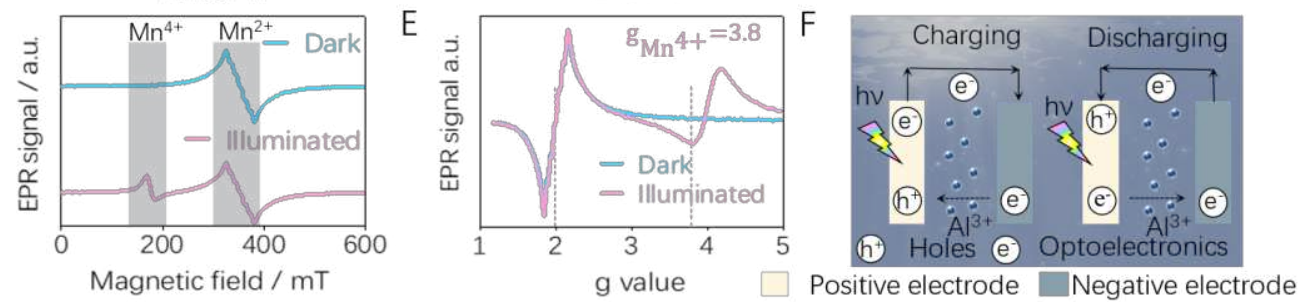

G
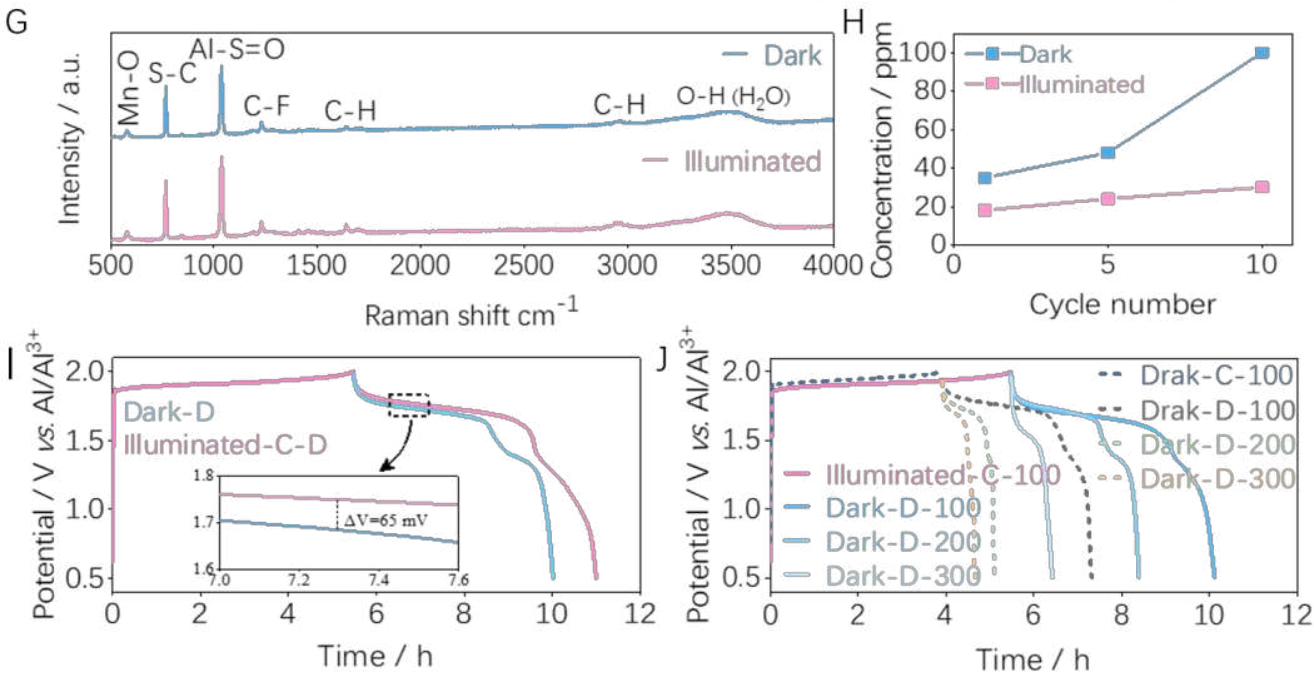

Fig. 4 Discussion of the photoelectrochemical coupling mechanism. (A) Intermittent

charge and discharge curves under dark and illuminated conditions at a current density of $100 \mathrm{~mA} \mathrm{~g}^{-1}$. (B) The photo-charging curves during the dark charging process, partial enlargement of (A). (C) Schematic diagram of the migration energy of light-excited electrons in $\alpha-\mathrm{MnO}_{2}$, where $\mathrm{Al}_{1}\left(\mathrm{MnO}_{2}\right)_{18}, \mathrm{Al}_{2}\left(\mathrm{MnO}_{2}\right)_{18}$, and $\mathrm{Al}_{2}\left(\mathrm{MnO}_{2}\right)_{18}$ represent the impurity levels. (D) Fully charged under light and then discharged in the dark at different current densities. Inset are the discharge curves in the same period under dark and illuminated conditions, respectively, exhibiting a potential difference of $65 \mathrm{mV}$. (E) Fully charged under light and then discharged at the same current density under dark and light, respectively. (F) EPR spectrum of a photo positive electrode discharged to 
$0.5 \mathrm{~V}$ under dark and illuminated conditions. (G) The Raman spectroscopy of the electrolyte after cycling in dark and illuminated conditions. (H) The concentration of $\mathrm{Mn}^{2+}$ in electrolyte at different cycles. (I) Corresponding g value distribution. (J) Schematic diagram of electron migration in the photoelectrochemical coupling process during charge and discharge. 
TOC Graphic

A high efficiency photo-regulation strategy has been proposed and established the stable photoelectrochemical coupling system to overcome the issues that the aqueous aluminum-manganese batteries are suffering. Consequently, the slow kinetics and undesired soluble $\mathrm{Mn}^{2+}$ have been significantly improved, thus achieving the photoelectrochemical batteries with high energy density and fast charge.

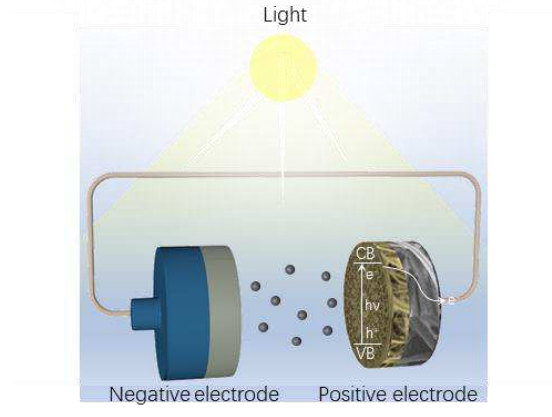




\section{Supplementary Files}

This is a list of supplementary files associated with this preprint. Click to download.

- SINC.docx 